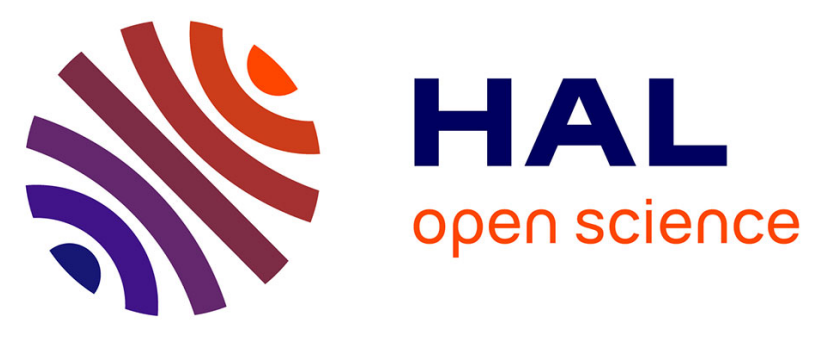

\title{
Combining photoinduced linkage isomerism and nonlinear optical properties in ruthenium nitrosyl complexes
}

\author{
Artem Mikhailov, Vedran Vuković, Christian Kijatkin, Emmanuel Wenger, \\ Mirco Imlau, Theo Woike, Gennadiy A Kostin, Dominik Schaniel
}

\section{To cite this version:}

Artem Mikhailov, Vedran Vuković, Christian Kijatkin, Emmanuel Wenger, Mirco Imlau, et al.. Combining photoinduced linkage isomerism and nonlinear optical properties in ruthenium nitrosyl complexes. Acta Crystallographica Section B : Structural Science, Crystal Engineering and Materials [2014-..], 2019, 75 (6), pp.1152-1163. 10.1107/S205252061901357X . hal-02386531

\section{HAL Id: hal-02386531 \\ https://hal.univ-lorraine.fr/hal-02386531}

Submitted on 29 Nov 2019

HAL is a multi-disciplinary open access archive for the deposit and dissemination of scientific research documents, whether they are published or not. The documents may come from teaching and research institutions in France or abroad, or from public or private research centers.
L'archive ouverte pluridisciplinaire HAL, est destinée au dépôt et à la diffusion de documents scientifiques de niveau recherche, publiés ou non, émanant des établissements d'enseignement et de recherche français ou étrangers, des laboratoires publics ou privés. 


\section{Combining photoinduced linkage isomerism and nonlinear optical properties in ruthenium nitrosyl complexes}

\section{Artem Mikhailov, Vedran Vuković, Christian Kijatkin, Emmanuel Wenger, Mirco Imlau, Theo Woike, Gennadiy Kostin and Dominik Schaniel}

Acta Cryst. (2019). B75, 1152-1163

\section{IUCr Journals CRYSTALLOGRAPHY JOURNALS ONLINE}

Copyright (C) International Union of Crystallography

Author(s) of this article may load this reprint on their own web site or institutional repository provided that this cover page is retained. Republication of this article or its storage in electronic databases other than as specified above is not permitted without prior permission in writing from the IUCr.

For further information see http://journals.iucr.org/services/authorrights.html 


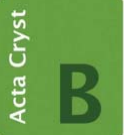

STRUCTURAL SCIENCE CRYSTAL ENGINEERING MATERIALS

ISSN 2052-5206

Received 11 July 2019

Accepted 4 October 2019

Edited by M. Dusek, Academy of Sciences of the Czech Republic, Czech Republic

Keywords: ruthenium nitrosyl; excited states; linkage isomers; nonlinear optical properties; photocrystallography.

CCDC reference: 1910521

Supporting information: this article has supporting information at journals.iucr.org/b

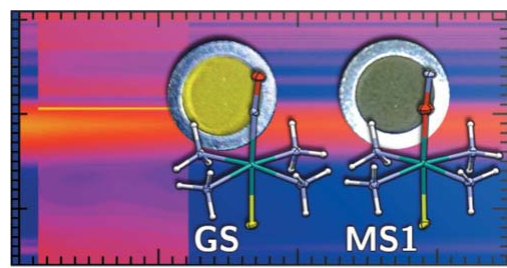

C 2019 International Union of Crystallography

\section{Combining photoinduced linkage isomerism and nonlinear optical properties in ruthenium nitrosyl complexes}

\author{
Artem Mikhailov, ${ }^{\mathrm{a}, \mathrm{b} *}$ Vedran Vuković, ${ }^{\mathrm{c}}$ Christian Kijatkin, ${ }^{\mathrm{d}, \mathrm{e}}$ Emmanuel Wenger, ${ }^{\mathrm{c}}$ \\ Mirco Imlau, ${ }^{\mathrm{d}}$ Theo Woike, ${ }^{\mathrm{c}}$ Gennadiy Kostin ${ }^{\mathrm{a}, \mathrm{b}}$ and Dominik Schaniel ${ }^{\mathrm{c} *}$
}

\begin{abstract}
aNikolaev Institute of Inorganic Chemistry, Siberian Branch of the Russian Academy of Sciences, 3 Acad. Lavrentiev Avenue, Novosibirsk 630090, Russian Federation, ${ }^{\mathbf{b}}$ Novosibirsk State University, 1 Pirogova Street, Novosibirsk 630090, Russian Federation, ' Université de Lorraine, CNRS, CRM2, UMR 7036, Nancy 54000, France, 'Department of Physics,

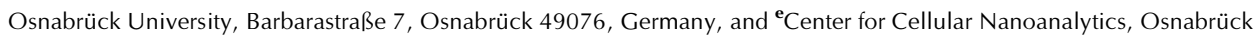
University, Barbarastraße 11, Osnabrück 49076, Germany. ${ }^{*}$ Correspondence e-mail: amikhailov@niic.nsc.ru, dominik.schaniel@univ-lorraine.fr
\end{abstract}

The complex trans-[RuNO $\left.\left(\mathrm{NH}_{3}\right)_{4} \mathrm{~F}\right] \mathrm{SiF}_{6}$ was synthesized in quantitative yield and the structure was characterized by $\mathrm{X}$-ray diffraction and spectroscopic methods. The complex crystallizes in the non-centrosymmetric space group $P n$. Hirshfeld surface analysis revealed that the dominant intermolecular interactions are of types $\mathrm{H} \cdots \mathrm{F}$ and $\mathrm{F} \cdots \mathrm{O}$, which are likely to be responsible for the packing of the molecules in a non-centrosymmetric structure. Irradiation with blue light leads to the formation of $\mathrm{Ru}-\mathrm{ON}$ (metastable state MS1) and $\mathrm{Ru}-\eta^{2}$-(NO) (metastable state MS2) bond isomers, as shown by IR and UV-Vis spectroscopy. The structural features of the MS1 isomer were elucidated by photocrystallography. The complex exhibits exceptionally good thermal stability of the metastable state MS1, such that it can be populated by light at 290-300 K, which is important for potential applications. The second harmonic (SH) emission can be generated by femtosecond-pulsed irradiation of the complex. The generated $\mathrm{SH}$ is rather efficient and stable under long-term exposure. Finally, since both metastable states and harmonic generation can be generated at room temperature, an attempt to drive the SH response by photoisomerization of the nitrosyl ligand was made and is discussed.

\section{Introduction}

Molecular materials based on a ruthenium metal centre exhibit a wide range of interesting optical properties. On the one hand, the absorption properties and refractive index of ruthenium nitrosyl complexes can be modified by the photogeneration of metastable states (Schaniel, Cormary et al., 2007; Schaniel, Imlau et al., 2007). These metastable states correspond to different types of coordination of the nitrosyl ligand to the $\mathrm{Ru}$ atom, i.e. nitrosyl linkage isomers (Coppens et al., 2002). In the ground state (GS) the nitrosyl ligand is coordinated through the $\mathrm{N}$ atom $(\mathrm{Ru}-\mathrm{NO})$, in metastable state 1 (MS1) through the $\mathrm{O}$ atom $(\mathrm{Ru}-\mathrm{ON})$ and in metastable state 2 (MS2) the coordination is of side-bond type [Ru- $\left.\eta^{2}-(\mathrm{NO})\right]$. An important feature is the possibility to switch reversibly between these three states with light, i.e. by using specific photon energies one can drive GS to MS1 $\left(h v_{1}\right)$ and MS1 to MS2 $\left(h v_{2}\right)$, while MS1 and MS2 can be transferred back to GS 
with a photon energy $h v_{3}$ (see Scheme 1 which shows photoswitching between linkage isomers in ruthenium nitrosyl).

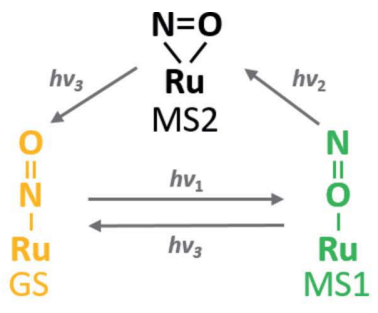

Scheme 1

On the other hand, ruthenium complexes exhibiting nonlinear optical (NLO) properties have been reported, allowing for e.g. second-harmonic ( $\mathrm{SH}$ ) generation (Asselberghs et al., 2004). In terms of even-order harmonic generation, the second-order polarizability $\beta$ [and equivalently the second-order susceptibility $\chi^{(2)}$ for solids] must be nonvanishing, which requires a non-centrosymmetric structure for solid bulk media (Clays \& Persoons, 1991; Roke \& Gonella, 2012). Third-harmonic (TH) generation, by contrast, is present in any space group and medium. Therefore, the ratio of the emission intensities of $\mathrm{SH}$ and $\mathrm{TH}$ can be exploited to gauge the NLO properties of new materials with respect to a standard (Kijatkin et al., 2017). Furthermore, a strong molecular dipole moment enhances the polarization effect and leads to even higher $\mathrm{SH}$ and $\mathrm{TH}$ intensities.

A tailored material for efficient harmonic generation may open up prospective applications, particularly in fields where significant emission signals are required, e.g. nonlinear imaging. Based on this, the design of potential molecular materials exhibiting NLO properties comes down to the creation of donor-acceptor interactions between the molecules within the material. Subsequently, a change in the donor-acceptor interaction by reduction of the acceptor or by oxidation of the donor may lead to a change in the NLO response with respect to the conversion efficiency. Redox switching can be triggered by chemical means, electrochemistry or light (Sakaguchi et al., 1989; Coe et al., 1999; Powell et al., 2003). Another interesting idea to control the NLO response was proposed in the work of Akl et al. (2013). Based on DFT calculations, those authors showed that the photoswitching of GS to MS1 in ruthenium nitrosyl complexes can induce a significant change in the hyperpolarizability parameter $\beta$ and might therefore be useful for NLO applications based on the second harmonic, such as for bioimaging (Urban et al., 2012; Su et al., 2017). However, there are certain experimental conditions to be satisfied in order to show that NLO properties can be controlled by photoswitching of nitrosyl linkage isomers. First, the complex needs to be crystallized in a non-centrosymmetric structure in order to produce SH generation in the solid state in the GS. Second, a sufficient amount of MS1 with a reasonable lifetime (ideally at room temperature) is needed in order to measure the change in NLO response upon generation of MS1.

In previous work we have shown that [RuNO(py) $\left.{ }_{4} \mathrm{~F}\right]\left(\mathrm{ClO}_{4}\right)_{2}$ exhibits the highest MS1 thermal stability and that MS1 can be photogenerated by blue light up to $230 \mathrm{~K}$ using a continuous-wave (CW) light source for irradiation, and even at room temperature when using pulsed laser irradiation (Kostin et al., 2018; Mikhailov et al., 2019). We also showed that the thermal stability of MS2 is essential for the roomtemperature generation of MS1, as the GS to MS1 excitation occurs via MS2 as an intermediate state (Mikhailov et al., 2019). Accordingly, the aim of the current study is to improve the thermal stability of MS1 and MS2, using fluorine as a transto-NO ligand, by the synthesis of trans- $\left[\mathrm{RuNO}\left(\mathrm{NH}_{3}\right)_{4} \mathrm{~F}\right] \mathrm{SiF}_{6}$, which crystallizes in a non-centrosymmetric space group. The structure of the complex is thoroughly analysed using X-ray diffraction (XRD) and $\mathrm{SH}$ generation. The properties and generation conditions of MS1 and MS2 are investigated by XRD, IR and UV-Vis spectroscopy and calorimetry. The NLO properties of GS are investigated in detail and an attempt is made to measure the changes induced by photogeneration of MS1 at room temperature.

\section{Experimental}

\subsection{Synthesis of trans-[RuNO $\left(\mathrm{NH}_{3}\right)_{4} \mathrm{~F}_{\mathrm{SiF}}$}

The synthesis of trans-[RuNO $\left.\left(\mathrm{NH}_{3}\right)_{4} \mathrm{~F}\right]^{2+}$ was performed in an analogous manner to that of the trans- $\left[\mathrm{RuNO}(\mathrm{py})_{4} \mathrm{~F}\right]^{2+}$ complex from our previous work (Kostin et al., 2018). trans$\left[\mathrm{RuNO}\left(\mathrm{NH}_{3}\right)_{4} \mathrm{OH}\right] \mathrm{Cl}_{2}(585 \mathrm{mg})$ was dissolved in concentrated $\mathrm{HF}(10 \mathrm{ml}, 40 \%)$ and heated in a closed polypropylene vessel for $30 \mathrm{~h}$ at $363 \mathrm{~K}$. The solution was then evaporated in air to dryness, resulting in a yellow residue. A few portions (3-5 ml) of diethyl ester were added to the solid in order to remove possible residual hydrofluoric acid. A yellow powder $(604 \mathrm{mg})$ with presumed formula trans-[RuNO $\left.\left(\mathrm{NH}_{3}\right)_{4} \mathrm{~F}\right]\left(\mathrm{H}_{n} \mathrm{~F}_{n+1}\right)_{2}$ was obtained. Single crystals of trans- $\left[\mathrm{RuNO}\left(\mathrm{NH}_{3}\right)_{4} \mathrm{~F}\right] \mathrm{SiF}_{6}$ were obtained by dissolving this product, trans-[RuNO$\left.\left(\mathrm{NH}_{3}\right)_{4} \mathrm{~F}\right]\left(\mathrm{H}_{n} \mathrm{~F}_{n+1}\right)$, in water in a glass tube. The yield of trans$\left[\mathrm{RuNO}\left(\mathrm{NH}_{3}\right)_{4} \mathrm{~F}\right] \mathrm{SiF}_{6}$ is $\sim 90 \%$.

Spectroscopic analysis: IR bands at room temperature $\left(\mathrm{cm}^{-1}\right): 3328,3228[v(\mathrm{C}-\mathrm{H})], 1899[v(\mathrm{NO})], 737,698[v(\mathrm{Si}-$ F)], 1668, 1608, 1566, 1324, 1302 and 870 (amine vibrations). Elemental analysis for $\mathrm{N}_{5} \mathrm{H}_{12} \mathrm{OF}_{7} \mathrm{Si}_{1} \mathrm{Ru}_{1}$, calculated/found (\%): H 3.4/3.6, N 19.4/19.7, F 36.9/37.2. The powder XRD pattern of the bulk sample corresponds to the theoretical pattern calculated from the crystal structure determined by single-crystal XRD (Fig. S1 in the supporting information).

\subsection{Single crystal $X$-ray diffraction}

One single crystal of trans-[RuNO $\left.\left(\mathrm{NH}_{3}\right)_{4} \mathrm{~F}\right] \mathrm{SiF}_{6}$ was selected and measured on a Rigaku Oxford Diffraction SuperNova four-circle diffractometer equipped with a molybdenum microfocus source (Mo $K \alpha, \lambda=0.71073 \AA$ ) and an Atlas CCD detector. An Oxford Cryosystems Cryostream nitrogen blower was used to maintain the sample temperature at $100 \mathrm{~K}$. After measuring the ground state (GS), the same single crystal was irradiated for $120 \mathrm{~min}$ by a $405 \mathrm{~nm} 360 \mathrm{~mW}$ LED at $100 \mathrm{~K}$ in order to measure the photoinduced state. The light of the LED was collimated using an anti-reflectioncoated aspheric condenser lens (COP1-A from Thorlabs), 
yielding a homogenous spot of about $20 \mathrm{~mm}$ diameter at a distance of $150 \mathrm{~mm}$. The crystal, of size $0.157 \times 0.104 \times$ $0.085 \mathrm{~mm}$, was centred on this spot and homogenous illumination was ensured by rotating the crystal by $90^{\circ}$ every $30 \mathrm{~min}$. The GS and photoinduced structures (corresponding to a mixture of GS and MS1) were solved using OLEX2 (Dolomanov et al., 2009) with the olex2.solve (Bourhis et al., 2015) structure solution program using charge flipping, and refined with the olex2.refine (Bourhis et al., 2015) refinement package using Gauss-Newton minimization. The GS structure of trans$\left[\mathrm{RuNO}\left(\mathrm{NH}_{3}\right)_{4} \mathrm{~F}\right] \mathrm{SiF}_{6}$ has been deposited with the CCDC with refcode 1910521. Data-collection and refinement parameters are given in Table S1.

\subsection{Hirshfeld surface analysis}

The Hirshfeld surfaces were calculated using Crystal Explorer (Hirshfeld, 1977; Spackman \& Jayatilaka, 2009; Turner et al., 2017). This program allows the normalized contact distance $d_{\text {norm }}$ to be mapped onto the generated Hirshfeld surface. It is customary to map $d_{\text {norm }}$ using a redwhite-blue scheme, where red denotes close intermolecular contacts (negative $d_{\text {norm }}$ ), blue denotes longer contacts (positive $d_{\text {norm }}$ ) and white denotes intermolecular contacts equal to the van der Waals radii of atoms in contact $\left(d_{\text {norm }}=0\right)$. It is possible to obtain two-dimensional plots (fingerprint plots) from the surfaces mapped with $d_{\text {norm }}$ values. Such plots are an invaluable asset in intermolecular interaction analysis since they serve as an executive summary of the quantity, nature and type of all intermolecular interactions at the same time.

\subsection{Powder X-ray diffraction}

Powder X-ray diffraction patterns were recorded using a PANalytical X'Pert PRO diffractometer equipped with a $\mathrm{Cu}$ $\mathrm{X}$-ray tube, a Ge(111) incident-beam monochromator $(\lambda=$ $1.5406 \AA$ A) and an X'Celerator detector.

\subsection{IR and UV-Vis spectroscopy}

IR spectroscopy measurements with irradiation were performed using a Nicolet 5700 FT-IR spectrometer with a resolution of $2 \mathrm{~cm}^{-1}$ in the range $400-4000 \mathrm{~cm}^{-1}$. The sample was ground, mixed with $\mathrm{KBr}$ and pressed into pellets. The $\mathrm{KBr}$ pellets were bonded by silver paste onto the cold finger of a closed-cycle cryostat (Oxford Optistat V01) and irradiated by an LED through $\mathrm{KBr}$ windows with light of different wavelengths in the range $300-940 \mathrm{~nm}$ and $10-400 \mathrm{~mW}$ optical power (Thorlabs L and LP series). The cryostat allows control of the temperature in the range 9-320 K.

UV-Vis spectra were recorded using transparent $\mathrm{KBr}$ pellets with the complex by a Varian CARY 4000 spectrometer. Transparent pellets were prepared as for the IR measurements. Low-temperature measurements were performed using the same cryostat as for the IR measurements, except that the $\mathrm{KBr}$ windows were exchanged for standard borosilicate glass windows. The baseline was measured using the same diaphragm with a $\mathrm{KBr}$ pellet, which can be mounted on the cryostat sample holder.

\subsection{Differential scanning calorimetry}

Differential scanning calorimetry (DSC) experiments were performed on a Mettler-Toledo DSC1 instrument equipped with a high-sensitivity DSC HSS8 sensor. The sample ( $\sim 5 \mathrm{mg}$ of the powder) was homogeneously spread in a standard aluminium crucible $(40 \mu \mathrm{l})$, such that it formed a thin layer covering the whole surface of the crucible.

For MS1 generation, irradiation of GS was performed at $143 \mathrm{~K}$ for $40 \mathrm{~min}$ through a glass window, using LED light at $405 \mathrm{~nm}$ (Thorlabs M405LP1-C1) and with a light intensity of $360 \mathrm{~mW}$. MS2 was generated under the same conditions by first irradiating at $405 \mathrm{~nm}$ for $40 \mathrm{~min}$ using $360 \mathrm{~mW}$, followed by irradiation at $940 \mathrm{~nm}$ and $200 \mathrm{~mW}$ (Thorlabs M940L3-C2) for $60 \mathrm{~min}$. To detect the enthalpy release during thermal relaxation of the metastable states, the sample was then heated from 143 to $353 \mathrm{~K}$ at a heating rate of $9 \mathrm{~K} \mathrm{~min}^{-1}$. As a reference measurement the sample was measured using the same protocol but without light irradiation. The experimental data were processed using the Netzsch Proteus analysis software.

\subsection{NLO properties}

Single crystals of trans-[RuNO$\left.\left(\mathrm{NH}_{3}\right)_{4} \mathrm{~F}\right] \mathrm{SiF}_{6}$ were ground in a mortar and pressed into a powder pellet as described by Kijatkin et al. (2017). For comparison, the same procedure was performed using magnesium-doped lithium niobate $\left(\mathrm{LiNbO}_{3}: \mathrm{Mg}\right.$ ) nanoparticles (mean particle size $d \simeq 90 \mathrm{~nm}$ ). Creating small-scale crystallites allows a loosening of the phase-matching conditions typically required for observing NLO signals. Spectrally tunable ultrashort laser pulses $(\tau \simeq$ $50 \mathrm{fs}$ ) at a pulse repetition rate of $1 \mathrm{kHz}$ were obtained by a regeneratively amplified femtosecond oscillator (Astrella $\mathrm{HE}+$ Coherent) equipped with an optical parametric amplifier (TOPAS Prime, Light Conversion). The incident laser pulses were directed onto the sample at a small angle of incidence $\left(\sim 30^{\circ}\right)$. Diffusely backscattered radiation was collected and spectrally resolved using a glass fibre placed perpendicular to the sample surface, which was attached to a combination of a spectrograph and a CCD camera (IsoPlane SCT320 and PIXIS:2KBUV, Princeton Instruments). Parallel population of the metastable states was achieved using a $405 \mathrm{~nm} \mathrm{CW}$ laser as a pump light source, irradiating the sample at a similar angle of incidence, thereby forming a symmetric beam geometry with respect to the sample surface. The intensity of both beams was regulated via neutral density filters. Backscattered pump radiation was attenuated before entering the spectrometer by a longpass filter (ZUL0422, Asahi Spectra).

\subsection{Pump-probe transient absorption spectroscopy}

The optical pump system consists of a Surelite II-10 NdYAG pulsed laser equipped with an optical parametric oscillator (OPO, model SLOPO+). The system delivers laser pulses of 3-5 ns duration at a frequency of $10 \mathrm{~Hz}$. The OPO was tunable in the range $400-700 \mathrm{~nm}$. The laser beam was directed onto the sample position (the complex in a $\mathrm{KBr}$ pellet) using a series of silver-coated mirrors and was precisely adjusted and 
focused through appropriate lenses $(f=100,200$ or $300 \mathrm{~mm})$ to illuminate the sample uniformly (i.e. the beam diameter was larger than the sample). For the transient absorption measurement, a CW laser at $532 \mathrm{~nm}$ wavelength was used to probe the light-induced absorption. The probe beam was focused by a lens $(f=300 \mathrm{~mm})$ onto the sample position such that the probe beam diameter was smaller than the pump beam diameter. Its intensity could be adjusted by a $\lambda / 2$ waveplate and a polariser from a few microwatts to a few milliwatts. The probe light was detected by a $200 \mathrm{MHz}$ Si-Pin diode from Femto Messtechnik GmbH. The voltage at the output of the detector was sampled using a $1.5 \mathrm{GHz}$ oscilloscope (LeCroy WavePro 715Zi). The trigger was obtained directly from the laser (with a delay of $10 \mathrm{~ns}$ with respect to the arrival of the pump beam on the sample position). In order to block the pump light $(410 \mathrm{~nm})$, two interference filters at $532 \mathrm{~nm}$ (width of $5 \mathrm{~nm}$ ) were mounted in front of the photodiode. The transient absorption signal was recorded in singleshot mode and averaged over 20 pump-probe cycles.

\subsection{Elemental analysis}

The elemental content of trans- $\left[\mathrm{RuNO}\left(\mathrm{NH}_{3}\right)_{4} \mathrm{~F}\right] \mathrm{SiF}_{6}(\mathrm{C}, \mathrm{H}$, $\mathrm{N})$ was determined using a Vario Microcube analyser. The fluorine content was determined after sample combustion by the Shoniger technique with an alkaline absorption liquid, by the spectrophotometric method with a lanthanum/alizarin complexone and by the potentiometric method with a fluorine-selective electrode. The discrepancy between the different methods did not exceed $0.1 \%$.

\section{Results and discussion}

\subsection{Synthesis and structural description}

Heating trans-[RuNO$\left.\left(\mathrm{NH}_{3}\right)_{4} \mathrm{OH}\right] \mathrm{Cl}_{2}$ with hydrofluoric acid results in the substitution of the $\mathrm{OH}^{-}$ligand by a fluoride anion. Conversion of the reaction was monitored by taking IR spectra of the resulting solid at different HF treatment times at
Table 1

Selected bond distances and angles $\left(\AA{ }^{\circ}\right)$ in trans- $\left[\mathrm{RuNO}\left(\mathrm{NH}_{3}\right)_{4} \mathrm{~F}\right] \mathrm{SiF}_{6}$.

\begin{tabular}{lllllr}
\hline Distance & \multicolumn{3}{l}{ Distance } & Angle & \\
\hline Ru1-N5 & $1.719(2)$ & Si1-F2 & $1.678(2)$ & Ru1-N5-O1 & $175.9(2)$ \\
N5-O1 & $1.144(2)$ & Si1-F3 & $1.713(2)$ & F1-Ru1-N5 & $177.2(1)$ \\
Ru1-F1 & $1.938(1)$ & Si1-F4 & $1.674(2)$ & N1-Ru1-F1 & $85.2(1)$ \\
Ru1-N1 & $2.091(2)$ & Si1-F5 & $1.681(2)$ & N2-Ru1-F1 & $87.1(1)$ \\
Ru1-N2 & $2.098(2)$ & Si1-F6 & $1.686(1)$ & N3-Ru1-F1 & $85.9(1)$ \\
Ru1-N3 & $2.092(2)$ & Si1-F7 & $1.661(1)$ & N4-Ru1-F1 & $85.9(1)$ \\
Ru1-N4 & $2.083(2)$ & & & & \\
\hline
\end{tabular}

$363 \mathrm{~K}$. After heating for $30 \mathrm{~h}$, the $v(\mathrm{NO})$ stretching vibration of the precursor complex $\left(1847 \mathrm{~cm}^{-1}\right)$ disappeared completely and a new $\nu$ (NO) band at $1875 \mathrm{~cm}^{-1}$ arose in the IR spectrum. In the next step, the resulting yellow crystalline powder with presumed formula trans- $\left[\mathrm{RuNO}\left(\mathrm{NH}_{3}\right)_{4} \mathrm{~F}\right]\left(\mathrm{H}_{n} \mathrm{~F}_{n+1}\right)_{2}$ was dissolved in water in a glass tube, leading to the formation of trans-[RuNO$\left.\left(\mathrm{NH}_{3}\right)_{4} \mathrm{~F}\right] \mathrm{SiF}_{6}$ single crystals. In an earlier report on the synthesis of trans-[RuNO$\left.\left(\mathrm{NH}_{3}\right)_{4} \mathrm{~F}\right]^{2+}$ (Sinitsyn et al., 1989), the precursor complexes trans-[RuNO $\left.\left(\mathrm{NH}_{3}\right)_{4} \mathrm{OH}\right]-$ $\left(\mathrm{NO}_{2}\right)_{2}$ or $\mathrm{K}_{2}\left[\mathrm{RuNOF}_{5}\right] \cdot \mathrm{H}_{2} \mathrm{O}$ were heated with molten $\mathrm{NH}_{4} \mathrm{HF}_{2}$ at $473 \mathrm{~K}$ for $\sim 1 \mathrm{~h}$ in a platinum melting pot. The treatment of the precursor in these aggressive conditions indicates the high thermal stability of the trans$\left[\mathrm{RuNO}\left(\mathrm{NH}_{3}\right)_{4} \mathrm{~F}\right]^{2+}$ cation. The final yield of the product was not mentioned in that report, but a few crystals of trans$\left[\mathrm{RuNO}\left(\mathrm{NH}_{3}\right)_{4} \mathrm{~F}\right] \mathrm{SiF}_{6}$ were obtained.

The complex trans-[RuNO $\left.\left(\mathrm{NH}_{3}\right)_{4} \mathrm{~F}\right] \mathrm{SiF}_{6}$ crystallizes in the non-centrosymmetric space group $P n$. The cell parameters are given in Table $\mathrm{S} 1$. The asymmetric unit contains two molecular ions, the $\left[\mathrm{RuNO}\left(\mathrm{NH}_{3}\right)_{4} \mathrm{~F}\right]^{2+}$ cation and the $\mathrm{SiF}_{6}{ }^{2-}$ anion, both in a distorted octahedral configuration [Fig. 1(a)]. The distances in the $\mathrm{F}-\mathrm{Ru}-\mathrm{N}-\mathrm{O}$ fragment are $\mathrm{Ru}-\mathrm{NO}=$ 1.719 (2) $\AA, \mathrm{N}-\mathrm{O}=1.144$ (2) $\AA$ and $\mathrm{Ru}-\mathrm{F}=1.938$ (1) (Table 1). The angles in the $\mathrm{F}-\mathrm{Ru}-\mathrm{N}-\mathrm{O}$ fragment are close to linear: $\mathrm{Ru} 1-\mathrm{N} 5-\mathrm{O} 1=175.9(2)^{\circ}$ and $\mathrm{F} 1-\mathrm{Ru} 1-\mathrm{N} 5=$ $177.2(1)^{\circ}$. While the bond distances $\mathrm{Ru}-\mathrm{N} 1$ and $\mathrm{Ru}-\mathrm{N} 3$ of the amine ligands trans to each other are equal [2.092 (2) $\AA$ ],

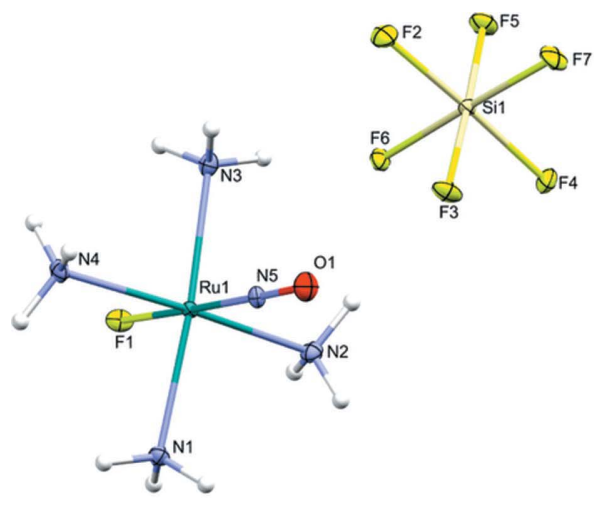

(a)

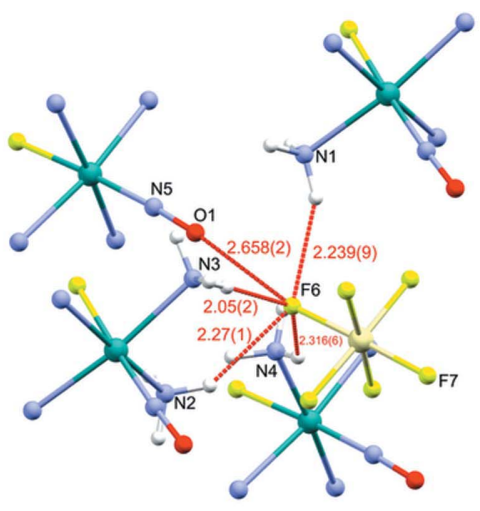

(b)

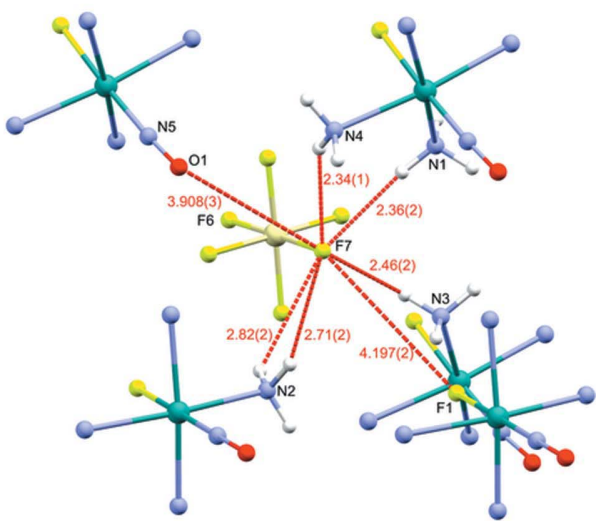

(c)

Figure 1

(a) The structure of the trans- $\left[\mathrm{RuNO}\left(\mathrm{NH}_{3}\right)_{4} \mathrm{~F}\right] \mathrm{SiF}_{6}$ complex. $(b)$ and $(c)$ The intermolecular contacts $(\AA)$ of F6 and F7. Ellipsoids in panel $(a)$ represent anisotropic displacement parameters at the $50 \%$ probability level. Spheres in panels $(b)$ and $(c)$ are of arbitrary radii. Most of the $\mathrm{H}$ atoms in panels $(b)$ and $(c)$ have been removed for clarity. 
the other two bond distances $\mathrm{Ru}-\mathrm{N} 2$ and $\mathrm{Ru}-\mathrm{N} 4$ exhibit a length difference of 0.015 (2) $\AA$. The difference is due to packing effects as a consequence of the different involvement of the amine ligands in hydrogen bonds with the F atoms of the hexafluorosilicate anion. These hydrogen bonds, alongside the halogen bonds, are also responsible for the almost-parallel alignment of the molecular dipoles, resulting in a noncentrosymmetric structure. In the following we discuss these important intermolecular interactions in more detail using Hirshfeld surface analysis.

The Hirshfeld surface analysis reveals an abundance of hydrogen bonds between the amino groups of the cation and the fluoride atoms of the $\mathrm{SiF}_{6}{ }^{2-}$ anion (Figs. 2 and S2). For the most part, individual bonds are reasonably strong (average $\mathrm{H}$. . F distance $2.170 \AA$, range 2.024-2.364 $\AA$, sum of van der Waals radii $2.66 \AA$, average $\mathrm{N}-\mathrm{H} \cdots \mathrm{F}$ angle $158.4^{\circ}$, range $\left.145.3-173.4^{\circ}\right)$. An average of two hydrogen bonds per $\mathrm{F}$ atom and several weaker contacts distort both octahedra (Table 1).

Reciprocal contacts are found on the Hirshfeld surface of the trans- $\left[\mathrm{RuNO}\left(\mathrm{NH}_{3}\right)_{4} \mathrm{~F}\right]^{2+}$ cation. One intense or two moderate red spots are noticeable near almost every $\mathrm{H}$ atom, indicating the existence of regular and bifurcated hydrogen bonds. The remaining $\mathrm{F}$ ligand, which is bound to $\mathrm{Ru}$, shows similar patterns of intermolecular contacts [Figs. 2 and S2(b)].

The nitrosyl ligand has a potential for both hydrogen and halogen bonds. There is a wealth of evidence for halogen bonds between $\mathrm{F} 6$ and $\mathrm{O} 1$ [Figs. 1(b), 1(c), S2 (c) and S2 $(f)$ ]. The F6 . . O 1 distance is $2.658 \AA$ (sum of van der Waals radii $2.96 \AA$ ), while the Si1-F6 . O 1 and F6 . O1 - N5 angles are $170.33^{\circ}$ and $171.50^{\circ}$, respectively. Furthermore, this halogen bond has a profound impact on the trans (to F6) ligand F7, which has the shortest $\mathrm{Si}-\mathrm{F} 7$ bond length of all the fluoride ligands. There is less evidence for hydrogen bonds between the amine ligands and O1 [Fig. S2 $(d)$ ]; these are weak hydrogen bonds with shallow angles at best.

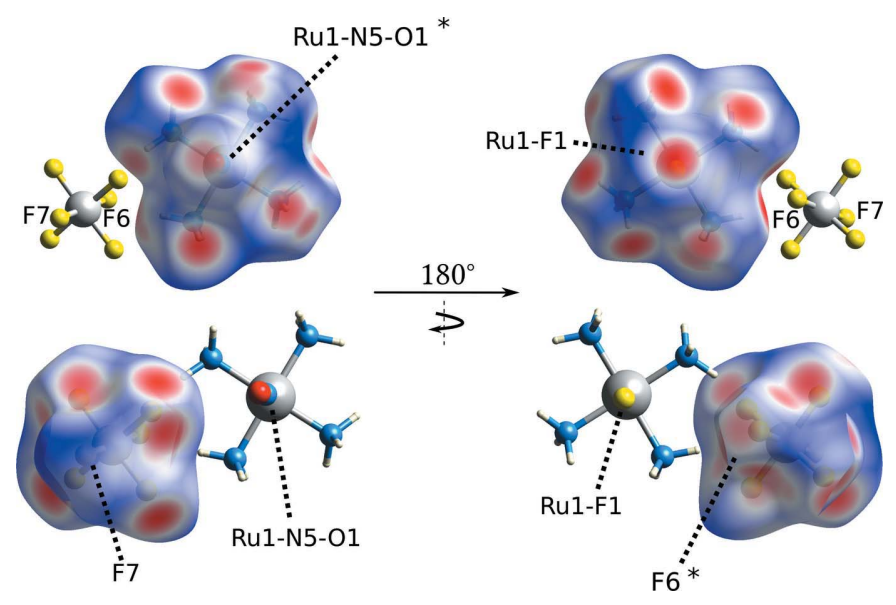

Figure 2

Three-dimensional Hirshfeld surface maps, coloured by $d_{\text {norm }}$ in the range 0.5-1.5 A. (Left) Front view and (right) back view. Regions coloured red and marked with an asterisk $(*)$ indicate F6 .. O 1 halogen bonds. Other red regions indicate $\mathrm{N}-\mathrm{H} \cdots \mathrm{F}$ bonds.

\subsection{Investigations of metastable states}

3.2.1. Spectroscopic and structural studies. IR spectroscopy is an extremely sensitive technique for detecting a bondlinkage change. The NO stretching vibration $v(\mathrm{NO})$ is a particularly useful fingerprint for the identification of nitrosyl linkage isomers (Mikhailov et al., 2019). Concerning the spectrum of GS, the $v(\mathrm{NO})$ band has a maximum at $1902 \mathrm{~cm}^{-1}$ with a shoulder at $1910 \mathrm{~cm}^{-1}$ at $10 \mathrm{~K}$. Light irradiation of GS in the range $300-500 \mathrm{~nm}$ generates MS1, characterized by its $v(\mathrm{ON})$ band with a maximum at $1769 \mathrm{~cm}^{-1}$ (Fig. 3). The $v(\mathrm{ON})$ band of MS1 exhibits a shift of $133 \mathrm{~cm}^{-1}$ to lower energy compared with the $v(\mathrm{NO})$ band of GS, which is typical for ruthenium nitrosyl compounds (Schaniel, Cormary et al., 2007; Kostin et al., 2015, 2017, 2018). The most efficient wavelengths for inducing the GS-to-MS1 transfer are in the range $405-420 \mathrm{~nm}$ (Fig. S3).

Further signatures of the MS1 isomer formation are the bands appearing at 577,532 and $466 \mathrm{~cm}^{-1}$, which we tentatively assign to the $v\left(\mathrm{Ru}-\mathrm{O}_{\mathrm{NO}}\right)$ stretching mode, the $\delta(\mathrm{Ru}-$ $\mathrm{O}-\mathrm{N})$ deformation mode and the $v(\mathrm{Ru}-\mathrm{F})$ stretching vibration, respectively. The appearance of these bands is accompanied by a decrease in the corresponding GS bands at 544,503 and $473 \mathrm{~cm}^{-1}$ (Fig. S4). The assignment is made based on the literature data (Weidemann et al., 1998). Again, the change in these low-energy vibrations upon MS1 formation is expected, as was shown for ruthenium nitrosyl complexes with trans-ligands $X=\mathrm{F}^{-}, \mathrm{OH}^{-}$(Kostin et al., 2015, 2017, 2018).

Concerning the vibrations of the amine groups, the appearance of new $\delta\left(\mathrm{NH}_{3}\right)$ bands of MS1 at 870 and $833 \mathrm{~cm}^{-1}$ and the corresponding decrease in the GS $\delta\left(\mathrm{NH}_{3}\right)$ bands at 876,854 and $848 \mathrm{~cm}^{-1}$, as well as perturbations of the $v(\mathrm{Ru}-$ $\mathrm{N}_{\mathrm{NH}_{3}}$ ) bands in the $1550-1700 \mathrm{~cm}^{-1}$ range after irradiation, are evidence again that the whole structure (axial and planar ligands of the octahedral complex) adapts to the NO rearrangement.

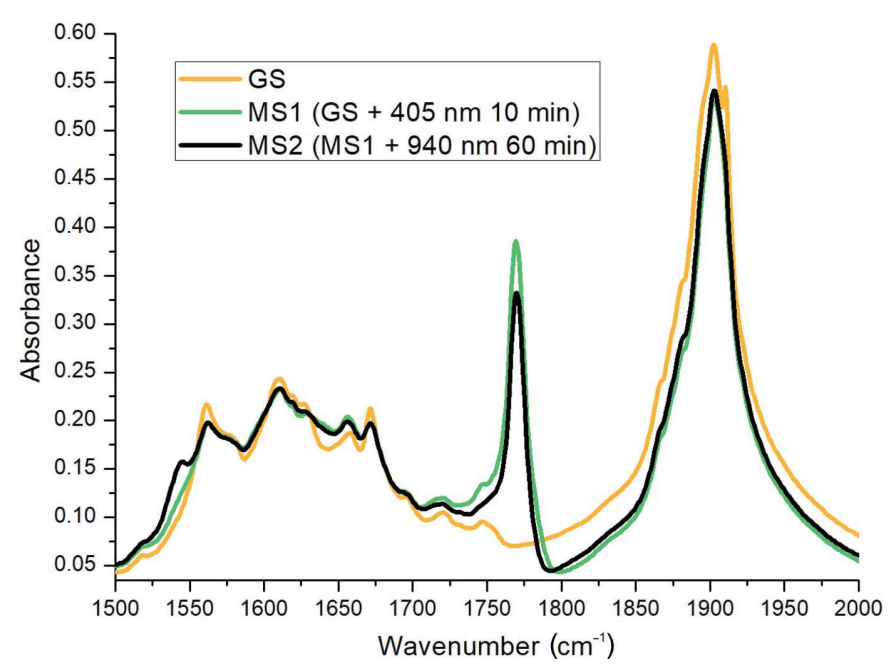

Figure 3

IR spectra at $10 \mathrm{~K}$ for the GS (orange line), GS+MS1 (green line) and GS+MS1+MS2 (black line). 
By irradiation of MS1 with $940 \mathrm{~nm}$ light it is possible to generate the MS2 isomer $\left[\mathrm{Ru}-\left(\eta^{2}-\mathrm{NO}\right)\right]$, i.e. irradiation in the near-IR induces the transfer of MS1 to MS2. The appearance of the MS2 isomer is characterized by the formation of the $v\left[\eta^{2}-(\mathrm{NO})\right]$ band with a maximum at $1545 \mathrm{~cm}^{-1}$. Compared with GS, the NO stretching vibration shifts to a significantly lower wavenumber by $357 \mathrm{~cm}^{-1}$, which again is typical for the MS2 isomer (Schaniel, Cormary et al., 2007; Kostin et al., 2015). Moreover, a new band with a maximum at $561 \mathrm{~cm}^{-1}$ is detected, most probably corresponding to the $\delta(\mathrm{Ru}-\mathrm{N}-\mathrm{O})$ deformation mode of MS2.

The populations of both MS are determined from the decrease in the area of the $v(\mathrm{NO})$ GS band and amount to $\sim 10 \%$ for MS1 and $\sim 3 \%$ for MS2. Irradiation of both MS1 and MS2 in the range 505-810 $\mathrm{nm}$ leads to a transfer back to GS. A summary of the wavelength dependence of the GS-MS transformations under light exposure is given in Fig. S5. As will be detailed below, the photogeneration of MS1 occurs through a two-step process via the second metastable state $\mathrm{MS} 2$, i.e. GS $\rightarrow \mathrm{MS} 2 \rightarrow \mathrm{MS} 1$, where MS2 is generated by a onephoton excitation. Any photon arriving within the lifetime of MS2 then induces the second step, MS2 $\rightarrow$ MS1, at the same wavelength.

In order to gain a better understanding of the population and depopulation processes of MS1 and MS2, the UV-Vis absorption spectra of trans- $\left[\mathrm{RuNO}\left(\mathrm{NH}_{3}\right)_{4} \mathrm{~F}\right] \mathrm{SiF}_{6}$ in $\mathrm{KBr}$ before and after light irradiation were measured at $100 \mathrm{~K}$ (Fig. 4). GS exhibits a strong absorption from the UV up to $500 \mathrm{~nm}$, whereas MS1 absorbs light over the whole measured range of 350-900 $\mathrm{nm}$ and has its lowest lying absorption band with a maximum around $550 \mathrm{~nm}$ (Fig. 4, inset). MS2, like MS1, absorbs over the entire measured range and exhibits an absorption maximum at $500 \mathrm{~nm}$. Within the GS $\rightarrow$ MS2 $\rightarrow$ MS1 generation scheme, GS can thus be excited to MS1 by irradiation in the range $405-420 \mathrm{~nm}$, due to the strong absorption bands of GS and MS2 and a comparable less efficient

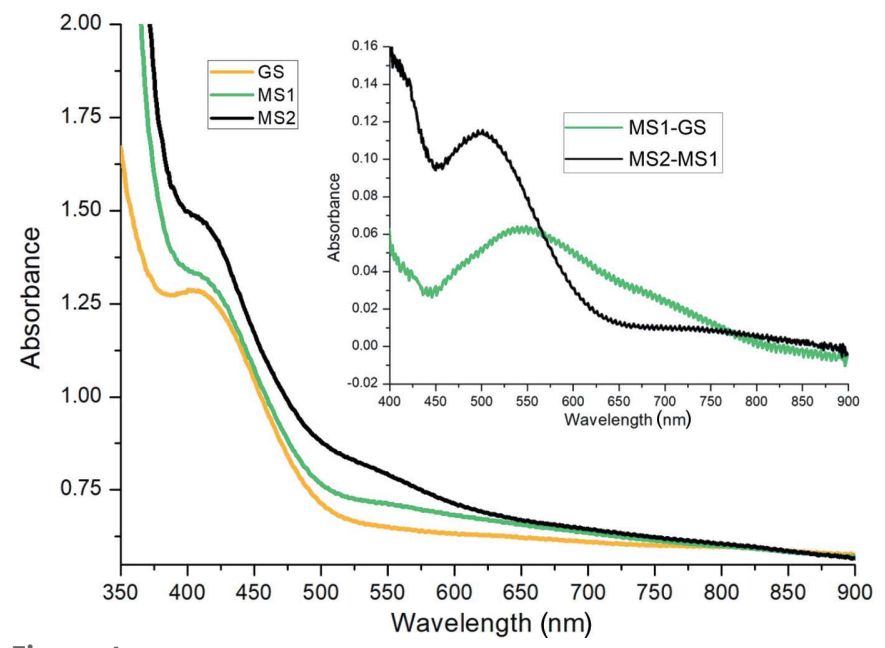

Figure 4

UV-Vis spectra for GS (orange line, before irradiation), GS+MS1 (green line, after 90 min of $405 \mathrm{~nm}$ irradiation of GS) and GS+MS1+MS2 (black line, after $60 \mathrm{~min}$ of subsequent $940 \mathrm{~nm}$ irradiation of MS1) at $100 \mathrm{~K}$. (Inset) MS1 and MS2 spectra after GS and MS1 subtraction, respectively. absorption of MS1 in this spectral range. Above $450 \mathrm{~nm}$, the MS1 absorption increases and, as a consequence, the cross section of the MS1-to-GS reaction increases, which results in a decrease in the population of MS1 in the case of excitation wavelengths of $450 \mathrm{~nm}$ and above (Fig. S3). Furthermore, since MS1 and MS2 exhibit significant absorption in the 450$810 \mathrm{~nm}$ spectral range, light irradiation in that region might lead to a transfer of MS1 and MS2 back to GS. IR exposure $(940 \mathrm{~nm}$ ) of MS1 results in the formation of MS2, but also in a slow back-transfer to GS, which indicates that the cross section of the MS1 $\rightarrow$ MS2 reaction in this spectral region is higher than for the MS1 $\rightarrow$ GS, MS2 $\rightarrow$ GS or MS2 $\rightarrow$ MS1 transformations.

While identification of the two linkage isomers MS1 and MS2 in nitrosyl compounds using IR spectroscopy is well established, a direct confirmation of the structural changes by $\mathrm{X}$-ray diffraction (XRD) is desirable. However, due to the relatively low populations of MS1 (10\%) and MS2 (3\%) and the significant spatial overlap of the different linkage-isomer configurations (especially GS and MS1), the structural analysis has to be performed with care. We restrict the photocrystallographic investigations to the case of MS1.

Initial model-independent evidence for NO isomerization is obtained by analysis of photodifference maps, an adequate tool for the study of structural changes even in the case of low populations (Schaniel et al., 2018). The same single crystal used for the GS structure determination was used for MS1 data collection after irradiation at $405 \mathrm{~nm}(360 \mathrm{~mW}$ LED for $120 \mathrm{~min})$. The crystal colour changes from bright yellow to pale yellow after light exposure (Fig. S6). The unit-cell parameters change from 6.7036 (4) to 6.7242 (4) $\AA$ and from 10.1296 (7) to 10.1347 (7) $\AA$ for $a$ and $c$, respectively, while $b$ changes only slightly from 7.4260 (4) to 7.4249 (4) $\AA$ (Table S1). Note that the $\mathrm{F}-\mathrm{Ru}-\mathrm{N}-\mathrm{O}$ axis is almost perpendicular to the $b$ axis, so the small increase in $a$ and $c$ might be expected from the lengthening of the $\mathrm{Ru}-\mathrm{O}$ distance in the $\mathrm{Ru}-\mathrm{ON}$ (MS1) bond compared with the $\mathrm{Ru}-\mathrm{N}$ distance in $\mathrm{Ru}-\mathrm{NO}$ (GS). The photodifference map is the Fourier transform of the difference between the structure factors of the photoexcited structure (GS+MS1) and the structure before irradiation (GS) (Schaniel et al., 2018). For the present case, the photodifference map (Fig. 5) is drawn in the $\mathrm{Ru}-\mathrm{N}-\mathrm{O}$ plane and shows a significant lack of electron density around atom O1. Near atom N5 some diffuse excess electron density is observed, but it is at the same level as the noise. This observation is typical for the case of a $180^{\circ} \mathrm{NO}$ isomerization, since the 'electron-rich' $\mathrm{O}$ atom in MS1 occupies the position of the $\mathrm{N}$ atom and vice versa. Therefore, in MS1 the electron density at the $\mathrm{O} 1$ position of GS is reduced, while at the N5 position it is increased. We also observe a change in electron density on the Ru position, which is due to a small displacement of Ru in MS1 compared with GS.

Having unambiguously established the presence of the MS1 isomer, we discuss in the following an approach to refine a structural model for MS1. In the first step, a structure refinement of GS+MS1 is performed using a single structural configuration to model the experimental data, one assuming a 
$100 \% \mathrm{Ru}-\mathrm{NO}$ configuration as for GS, and one using a $100 \%$ $\mathrm{Ru}-\mathrm{ON}$ configuration as one might expect if the GS to MS1 conversion were complete. Atomic distances for the structures obtained after these two refinements are shown in Table 2. Regardless of the model used ( $\mathrm{Ru}-\mathrm{NO}$ or $\mathrm{Ru}-\mathrm{ON})$, the distance between $\mathrm{Ru}$ and the nitrosyl ligand increases by $0.02-$ $0.03 \AA$. Residual electron-density maps of both structure refinements are shown in Fig. S7. For the model assuming the GS Ru-NO configuration, no excess or lack of electron density around the nitrosyl ligand is observed. In the case of the model with an $\mathrm{Ru}-\mathrm{ON}$ configuration, significant residuals are observed. There is a lack of electron density near the $\mathrm{N}$ atom and an excess of electron density around the $\mathrm{O}$ atom of the nitrosyl ligand. The lack of electron density indicates that the $\mathrm{N}$ atom should be substituted by a more electron-rich atom, while the excess of electron density indicates that the $\mathrm{O}$ atom should be substituted by a less electron-rich atom. Since the model with $\mathrm{Ru}-\mathrm{NO}$ yields a better fit of the GS+MS1 structure and the elongation of the $\mathrm{Ru}-(\mathrm{NO})$ distance is only 0.02-0.03 $\AA$, we can conclude that the population of the $\mathrm{Ru}-$ $\mathrm{ON}$ isomer is significantly lower than that of the $\mathrm{Ru}-\mathrm{NO}$ isomer, in agreement with the spectroscopic results. For comparison, in the $\mathrm{K}_{2}\left[\mathrm{RuNO}\left(\mathrm{NO}_{2}\right)_{4} \mathrm{OH}\right], \quad\left[\mathrm{RuNO}(\mathrm{py})_{2}\right.$ $\left.\left(\mathrm{NO}_{2}\right)_{2} \mathrm{OH}\right], \quad\left[\mathrm{RuNO}(\mathrm{py})_{4} \mathrm{Cl}\right]\left(\mathrm{PF}_{6}\right)_{2} \cdot 0.5 \mathrm{H}_{2} \mathrm{O}$ and [RuNO(py $\left.)_{4} \mathrm{~F}\right](\mathrm{ClO} 4)_{2}$ ruthenium nitrosyl complexes, the elongation of the Ru-(NO) bond distance from GS to MS1 was 0.1 (1) (Fomitchev \& Coppens, 1996; Cormary et al., 2009; Kostin et al., 2015; Mikhailov et al., 2019).

In the second step, the excited structure is modelled using a split model for the NO group, i.e. a mixture of the two configurations $\mathrm{Ru}-\mathrm{ON}$ and $\mathrm{Ru}-\mathrm{NO}$ (Table 2). In the light of

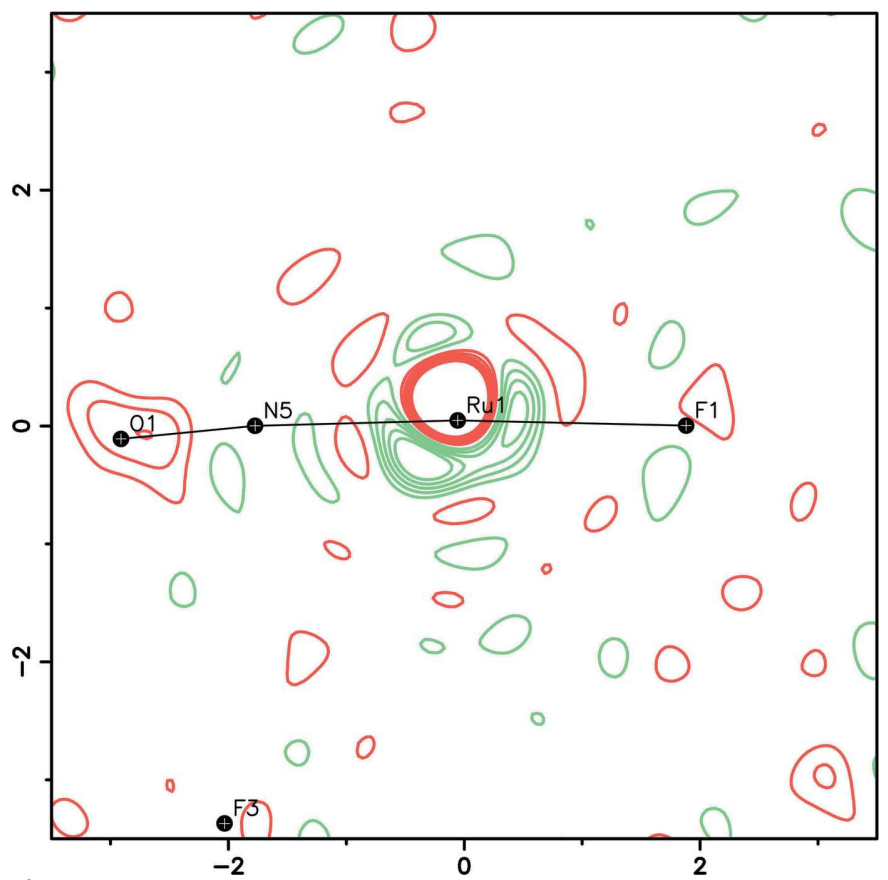

Figure 5

A two-dimensional photodifference map between the photoinduced state (GS+MS1) and GS. Residual electron-density contours are 0.35 e $\AA^{-3}$. A lack of electron density is shown by red contours and an excess by green contours.
Table 2

Selected bond distances and angles $\left(\AA,^{\circ}\right)$ for GS and for the three models of GS+MS1.

\begin{tabular}{|c|c|c|c|c|}
\hline \multirow[b]{3}{*}{ Distance/angle } & \multirow[b]{2}{*}{ GS } & \multicolumn{3}{|l|}{ GS+MS1 } \\
\hline & & GS $(100 \%)$ & MS1 (100\%) & $\begin{array}{l}\text { GS/MS1 }(80 / 20 \%) \\
\text { split model }\end{array}$ \\
\hline & $\mathrm{Ru}-\mathrm{NO}$ & $\mathrm{Ru}-\mathrm{NO}$ & $\mathrm{Ru}-\mathrm{ON}$ & $\mathrm{Ru}-\mathrm{NO} / \mathrm{Ru}-\mathrm{ON}$ \\
\hline Ru1-N5 & $1.719(2)$ & $1.740(2)$ & & $1.731(3)$ \\
\hline Ru1-O1 & & & $1.747(2)$ & $1.789(5)$ \\
\hline N5-O1 & $1.144(2)$ & $1.148(2)$ & $1.141(2)$ & $1.157(4) / 1.13(1)$ \\
\hline $\mathrm{Ru} 1-\mathrm{F} 1$ & $1.938(1)$ & $1.936(1)$ & $1.939(2)$ & $1.936(1)$ \\
\hline $\mathrm{Ru} 1-\mathrm{N} 1$ & $2.091(2)$ & $2.090(3)$ & $2.093(3)$ & $2.091(2)$ \\
\hline $\mathrm{Ru} 1-\mathrm{N} 2$ & $2.098(2)$ & $2.103(3)$ & $2.099(3)$ & $2.102(3)$ \\
\hline $\mathrm{Ru} 1-\mathrm{N} 3$ & $2.092(2)$ & $2.092(3)$ & $2.088(3)$ & $2.092(3)$ \\
\hline $\mathrm{Ru} 1-\mathrm{N} 4$ & $2.083(2)$ & $2.082(3)$ & $2.083(3)$ & $2.083(3)$ \\
\hline Si1-F2 & $1.678(2)$ & $1.684(3)$ & $1.686(3)$ & $1.684(3)$ \\
\hline Si1-F3 & $1.713(2)$ & $1.712(2)$ & $1.713(2)$ & $1.712(2)$ \\
\hline Si1-F4 & $1.674(2)$ & 1.677 (3) & $1.676(3)$ & $1.677(3)$ \\
\hline Si1-F5 & $1.681(2)$ & $1.686(2)$ & $1.684(2)$ & $1.686(2)$ \\
\hline Si1-F6 & $1.686(1)$ & $1.690(2)$ & $1.687(2)$ & $1.690(2)$ \\
\hline Si1-F7 & $1.661(1)$ & $1.664(2)$ & $1.669(2)$ & $1.664(2)$ \\
\hline $\mathrm{Ru} 1-\mathrm{N} 5-\mathrm{O} 1$ & $175.9(2)$ & $175.7(2)$ & & $173.0(4)$ \\
\hline $\mathrm{Ru} 1-\mathrm{O} 1-\mathrm{N} 5$ & & & $175.4(2)$ & $165(1)$ \\
\hline $\mathrm{F} 1-\mathrm{Ru} 1-\mathrm{N} 5$ & $177.2(1)$ & $177.2(1)$ & & $177.9(2)$ \\
\hline $\mathrm{F} 1-\mathrm{Ru} 1-\mathrm{O} 1$ & & & $176.8(1)$ & $173.9(3)$ \\
\hline $\mathrm{N} 1-\mathrm{Ru} 1-\mathrm{F} 1$ & $85.2(1)$ & $85.4(1)$ & $85.8(1)$ & $86.5(1)$ \\
\hline $\mathrm{N} 2-\mathrm{Ru} 1-\mathrm{F} 1$ & $87.1(1)$ & $87.5(1)$ & $87.5(1)$ & $87.5(1)$ \\
\hline $\mathrm{N} 3-\mathrm{Ru} 1-\mathrm{F} 1$ & 85.9 (1) & $86.6(1)$ & $86.4(1)$ & $85.5(1)$ \\
\hline $\mathrm{N} 4-\mathrm{Ru} 1-\mathrm{F} 1$ & 85.9 (1) & $86.1(1)$ & $86.1(1)$ & $86.1(1)$ \\
\hline
\end{tabular}

our conclusions above and the fact that the population of MS1 is $10 \%$ from the IR measurements, we have constrained the occupancy of MS1 to 20\%. In the case of a refinement with less than $20 \%$ of MS1, the MS1 displacement parameters of the nitrosyl atoms become non-positive definite even when using an isotropic refinement. Using this constrained model, we obtain an $\mathrm{Ru}-\mathrm{ON}$ bond length of 1.789 (5) $\AA$, which is 0.07 (1) $\AA$ longer than in the GS structure. The $\mathrm{Ru}-\mathrm{O}-\mathrm{N}$ angle changes to $165(1)^{\circ}$. All other bond distances in the GS+MS1 structure are the same as in GS to within the limit of error. Since the population of the $\mathrm{Ru}-\mathrm{ON}$ isomer is only 10 $20 \%$, the accuracy of the structural parameters obtained from the refinement of the structure of MS1 is limited, as discussed in the literature (Cormary et al., 2009; Kostin et al., 2015; Mikhailov et al., 2019). For example, the largest change in the bond lengths after blue-light irradiation is the elongation of the $\mathrm{Ru}-\mathrm{ON}$ bond by 0.1 (1) $\AA$, which can be unambiguously refined only in the case of high populations of the $\mathrm{Ru}-\mathrm{ON}$ state. In case of an MS1 population of less than $50 \%$, the structural parameters of MS1 in the presence of GS should be interpreted with care. Simulations showed that typical errors on the Ru1-O1 distance are of the order of $0.03 \AA$. For an indepth discussion of the correlation between population and structural and anisotropic displacement parameters we refer the reader to the work of Cormary et al. (2009). All in all, we can conclude that MS1 of trans- $\left[\mathrm{RuNO}\left(\mathrm{NH}_{3}\right)_{4} \mathrm{~F}\right] \mathrm{SiF}_{6}$ represents the typical structural behaviour of the isonitrosyl isomer in the nitrosyl ruthenium family.

3.2.2. Thermal stability of metastable states. For potential applications the thermal stability of MS1 and MS2 is essential. We determined the energy barriers of the MS1-to-GS and 
Table 3

Kinetic parameters for the MS1 $\rightarrow$ GS reaction.

\begin{tabular}{|c|c|c|c|c|c|}
\hline Trans complex & $E_{\mathrm{a}}(\mathrm{eV})$ & $k_{0}\left(\mathrm{~s}^{-1}\right)$ & $T_{\mathrm{d}}(\mathrm{K})$ & $T_{\max \text { of } \operatorname{DSC}^{\dagger}(\mathrm{K})}$ & Reference \\
\hline$\left[\mathrm{RuNO}(\mathrm{en})_{2} \mathrm{Br}\right] \mathrm{Cl}_{2}$ & 0.53 & $5.2 \times 10^{8}$ & 229 & $\approx 250 \ddagger$ & Ookubo et al. (1996) \\
\hline$\left[\mathrm{RuNO}(\mathrm{en})_{2} \mathrm{Cl}\right] \mathrm{Cl}_{2}$ & 0.69 & $1.7 \times 10^{11}$ & 246 & $\approx 270 \ddagger$ & Ookubo et al. (1996) \\
\hline$\left[\mathrm{RuNO}\left(\mathrm{NH}_{3}\right)_{4} \mathrm{OH}\right] \mathrm{Cl}_{2}$ & 0.91 & $6 \times 10^{14}$ & 258 & $\approx 280$ & Schaniel et al. (2005) \\
\hline$\left[\mathrm{RuNO}(\mathrm{py})_{4} \mathrm{Br}\right]\left(\mathrm{PF}_{6}\right)_{2}$ & & & & $\approx 210$ & Coppens et al. (2002) \\
\hline$\left[\mathrm{RuNO}(\mathrm{py})_{4} \mathrm{OH}\right]\left(\mathrm{PF}_{6}\right)_{2}$ & & & & $\approx 220$ & Coppens et al. (2002) \\
\hline$\left[\mathrm{RuNO}(\mathrm{py})_{4} \mathrm{Cl}\right]\left(\mathrm{PF}_{6}\right)_{2}$ & 0.70 & $2 \times 10^{12}$ & 231 & $\approx 250$ & Schaniel et al. (2007) \\
\hline$\left[\mathrm{RuNO}(\mathrm{py})_{4} \mathrm{~F}\right]\left(\mathrm{ClO}_{4}\right)_{2}$ & 1.02 & $7.9 \times 10^{14}$ & 289 & $\approx 310$ & Kostin et al. (2018) \\
\hline
\end{tabular}

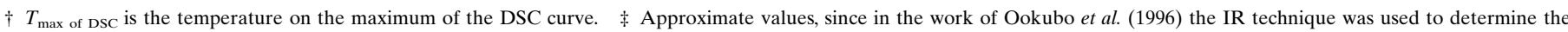

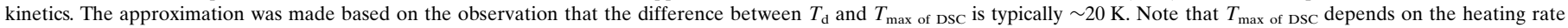
(Kostin et al., 2015).

MS2-to-GS reactions using DSC. The kinetic parameters of the exothermic reactions (activation energies $E_{\mathrm{a}}$ and frequency factors $k_{0}$ ) were calculated using the first-order kinetic equation

$$
\mathrm{d} H / \mathrm{d} t=H_{\text {tot }}(1-\alpha) k_{0} \exp \left[-E_{\mathrm{a}} /(R T)\right],
$$

where $\mathrm{d} H / \mathrm{d} t$ is the heat-flow rate, $H_{\text {tot }}$ is the total reaction enthalpy, $\alpha$ is the conversion, $R$ is the universal gas constant and $T$ is the temperature. The DSC curves are shown in Fig. S8. According to the fit, $E_{\mathrm{a}}$ and $\log k_{0}$ for the $\mathrm{MS} 1 \rightarrow \mathrm{GS}$ and $\mathrm{MS} 2 \rightarrow \mathrm{GS}$ reactions are $97.4(3) \mathrm{kJ} \mathrm{mol}^{-1}$ and 14.42 (4), and 59.0 (5) $\mathrm{kJ} \mathrm{mol}^{-1}$ and 12.0 (1), respectively.

A useful parameter to compare the thermal stability of MS of different complexes is the so-called decay temperature $T_{\mathrm{d}}$ (Morioka et al., 2000), which is the temperature calculated for a value of the rate constant of $k=10^{-3} \mathrm{~s}^{-1}$ from the Arrhenius law,

$$
k=k_{0} \exp \left[-E_{\mathrm{a}} /(R T)\right] .
$$

The $T_{\mathrm{d}}$ values for MS1 and MS2 are 292 and $206 \mathrm{~K}$, respectively. The $T_{\mathrm{d}}$ for MS1 in trans-[RuNO$\left.\left(\mathrm{NH}_{3}\right)_{4} \mathrm{~F}\right] \mathrm{SiF}_{6}$ is the highest decay temperature known up to now (see Table 3), thereby confirming that the fluorine ligand trans to NO has a positive effect on the thermal stability (Yamaletdinov \& Zilberberg, 2017; Kostin et al., 2018). In order to illustrate the influence of the trans-to-NO ligand on the thermal stability of MS1, Table 3 summarizes the kinetic parameters of the $\mathrm{MS} 1 \rightarrow \mathrm{GS}$ reaction for a series of complexes with the same ligand environment $\left(\mathrm{NH}_{3}\right.$, ethylenediamine and pyridine in the equatorial position) and charge. Earlier, the influence of the trans-to-NO ligand in trans- $\left[\mathrm{RuNO}\left(\mathrm{NH}_{3}\right)_{4} \mathrm{X}\right]^{2+}$ on the activation energy of the MS1 $\rightarrow$ GS reaction was investigated by density functional theory (DFT) calculations (Yamaletdinov \& Zilberberg, 2017). According to that work, $E_{\mathrm{a}}$ increases in the sequence $X=\mathrm{SH}^{-}, \mathrm{OH}^{-}, \mathrm{Cl}^{-}, \mathrm{F}^{-}$and equals $0.66,0.97,1.04$ and $1.24 \mathrm{eV}$, respectively. A discussion of the difference between the calculations and the experimental data is beyond the scope of the present study, but one can state that the same trends are observed by both approaches and the complexes with $X=\mathrm{F}^{-}$show the highest $E_{\mathrm{a}}$ and $T_{\mathrm{d}}$ for the $\mathrm{MS} 1 \rightarrow$ GS transformation.

3.2.3. Generation of MS1 and MS2 at room temperature. The temperature at which MS1 can be populated depends strongly on the MS2 lifetime due to the two-step isomerization mechanism GS $\rightarrow \mathrm{MS} 2 \rightarrow \mathrm{MS} 1$, i.e. increasing the lifetime of MS2 will lead to an increase in the temperature at which MS1 can be populated (Mikhailov et al., 2019). Since trans$\left[\mathrm{RuNO}\left(\mathrm{NH}_{3}\right)_{4} \mathrm{~F}\right] \mathrm{SiF}_{6}$ and trans-[RuNO$\left.(\mathrm{py})_{4} \mathrm{~F}\right]\left(\mathrm{ClO}_{4}\right)_{2}$ exhibit almost the same $T_{\mathrm{d}}$ of MS1 (292 and $289 \mathrm{~K}$, respectively) but different $T_{\mathrm{d}}$ of MS2 (206 and $185 \mathrm{~K}$, respectively; Kostin et al., 2018), we expect to increase the temperature at which MS1 can be populated using CW light sources like LEDs (230 K for trans-[RuNO(py $\left.)_{4} \mathrm{~F}\right]\left(\mathrm{ClO}_{4}\right)_{2}$; Mikhailov et al., 2019).

The IR spectra of the trans- $\left[\mathrm{RuNO}\left(\mathrm{NH}_{3}\right)_{4} \mathrm{~F}\right] \mathrm{SiF}_{6}$ complex recorded after $15 \mathrm{~min}$ of $405 \mathrm{~nm}$ irradiation (LED, $360 \mathrm{~mW}$ ) at temperatures of 290, 295 and $300 \mathrm{~K}$ are shown in Fig. 6. After irradiation of GS at these temperatures, a band with a maximum at $1766 \mathrm{~cm}^{-1}$ is clearly visible in the spectrum, corresponding to the $v(\mathrm{ON})$ band characteristic of the MS1 isomer. The decay of this band at 300, 295 and $290 \mathrm{~K}$ occurs over $\sim 300, \sim 700$ and $\sim 1300 \mathrm{~s}$, respectively, which correspond to the lifetimes of MS1 calculated from the DSC measurements (353, 684 and $1357 \mathrm{~s}$ at 300, 295 and $290 \mathrm{~K}$, respectively). The decay of the MS1 band is shown as an example for the measurement at $290 \mathrm{~K}$ in Fig. S9, together with a mono-

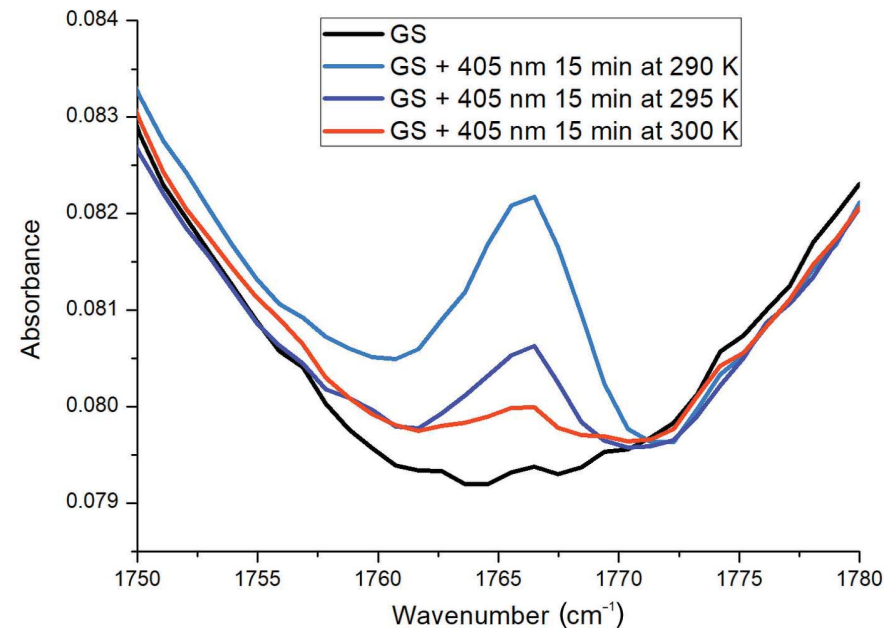

Figure 6

The generation of the MS1 isomer by $15 \mathrm{~min}$ of $405 \mathrm{~nm} 360 \mathrm{~mW}$ irradiation at different temperatures: $290 \mathrm{~K}$ (blue line), $295 \mathrm{~K}$ (purple line) and $300 \mathrm{~K}$ (red line). The populations of MS1 at these temperatures are $0.5,0.4$ and $0.2 \%$, respectively. 
exponential fit of the relaxation. Thus, for trans$\left[\mathrm{RuNO}\left(\mathrm{NH}_{3}\right)_{4} \mathrm{~F}\right] \mathrm{SiF}_{6}$, the photogeneration of MS1 using CW light sources is possible even at $300 \mathrm{~K}$, which is a necessary prerequisite in view of potential applications, and an important improvement with respect to trans-[RuNO$\left.(\mathrm{py})_{4} \mathrm{~F}\right]-$ $\left(\mathrm{ClO}_{4}\right)_{2}$, where MS1 photogeneration at $300 \mathrm{~K}$ was only achieved by pulsed-laser irradiation (Mikhailov et al., 2019). This improvement is due to the increase in the activation energy of the $\mathrm{MS} 2 \rightarrow \mathrm{GS}$ reaction by $11.5 \mathrm{~kJ} \mathrm{~mol}^{-1}(0.1 \mathrm{eV})$ from trans $-\left[\mathrm{RuNO}(\mathrm{py})_{4} \mathrm{~F}\right]\left(\mathrm{ClO}_{4}\right)_{2} \quad\left[E_{\mathrm{a}}(\mathrm{MS} 2 \rightarrow \mathrm{GS})=\right.$ $46.4 \mathrm{~kJ} \mathrm{~mol}^{-1}$; Kostin et al., 2018; Mikhailov et al., 2019] to trans- $\left[\mathrm{RuNO}\left(\mathrm{NH}_{3}\right)_{4} \mathrm{~F}\right] \mathrm{SiF}_{6}\left[E_{\mathrm{a}}(\mathrm{MS} 2 \rightarrow \mathrm{GS})=59.0 \mathrm{~kJ} \mathrm{~mol}^{-1}\right]$. The increase in activation energy leads to an increased MS2 lifetime, which results in a higher probability of the $\mathrm{MS} 2 \rightarrow \mathrm{MS} 1$ reaction via the $\mathrm{GS} \rightarrow \mathrm{MS} 2 \rightarrow \mathrm{MS} 1$ mechanism.

Further support for this two-step mechanism of isomerization is obtained from transient nanosecond pump-CW probe spectroscopy at room temperature. The temporal decay of the light-induced absorption after pumping with a $410 \mathrm{~nm}$ laser pulse is shown in Fig. S10. The absorption decay is fitted by mono-exponential kinetics and characterized by a 13 (1) ms lifetime, corresponding to the MS2 state and its thermal decay process, which is in agreement with the calculated MS2 lifetime at $\simeq 300 \mathrm{~K}$. These findings show, again, that after excitation of the complex with a single laser pulse a small but measurable amount of the MS2 state can be populated, while long-term irradiation with a $405 \mathrm{~nm}$ LED allows the generation of MS1 by a sequential two-step photon absorption from GS to MS2 to MS1.

\subsection{NLO properties - generation of the second and third harmonics}

Our structural investigations of trans- $\left[\mathrm{RuNO}\left(\mathrm{NH}_{3}\right)_{4} \mathrm{~F}\right] \mathrm{SiF}_{6}$ confirmed that it crystallizes in a non-centrosymmetric space group. The lack of an inversion centre should enable evenorder harmonic generation, in particular second-harmonic (SH) generation. Using femtosecond pulses in the near-IR with peak intensities of the order of $3 \times 10^{14} \mathrm{~W} \mathrm{~m}^{-2}$ (a timeaveraged power of approximately $0.8 \mathrm{~W} \mathrm{~cm}^{-2}$ ) reveals a remarkable harmonic emission, as shown in Fig. 7.

Because of the pronounced absorption in the blue spectral range, the fundamental wavelength was tuned between $\lambda=$ 1400 and $1560 \mathrm{~nm}$ in order to shift both second and third harmonics further into the transmission window (see Fig. 4) for an unobstructed generation of harmonics, in particular of the third harmonic. Regardless of the selected wavelength, the observed SH emission intensity becomes of notable interest, exceeding that of a standard NLO material such as lithium niobate (LN) by a factor of approximately three. Simultaneously, the TH signal of trans- $\left[\mathrm{RuNO}\left(\mathrm{NH}_{3}\right)_{4} \mathrm{~F}\right] \mathrm{SiF}_{6}$ is up to an order of magnitude weaker than for $\mathrm{LN}$, which can be explained by an absorption band in the range of 400-550 nm. As a result, the harmonic ratio $f_{\mathrm{R}}$, describing the polarity of the material and providing an accessible quantity for the characterization of second-order nonlinearities (Kijatkin et al.,
2017; Athmani et al., 2019), is considerably higher for the ruthenium complex compared with $\mathrm{LN}$.

In this context, it is worth discussing the potential origins of the remarkable nonlinear emission. The overall amplitude of the signal may partially stem from the different crystallite size, as it has been shown that the $\mathrm{SH}$ signal is sensitive to the particle volume (Knabe et al., 2012; Kim et al., 2013). However, this explanation does not fully suffice in elucidating the relative weakness of the $\mathrm{TH}$ peak, considering that, at the present length scales, it should increase with the particle volume as well. Because the second-order nonlinear susceptibility $\chi^{(2)}$ is composed of the individual hyperpolarizabilities $\beta_{i}$ of the building blocks inside a volume element of the crystal (which likewise can be applied to higher orders; Chemla, 1980), a thorough look is required on the molecular level.

According to Akl et al. (2013), the molecular hyperpolarizabilities calculated from DFT for $[\mathrm{Ru}(\mathrm{NO})(R$-terpyridine $\left.) \mathrm{Cl}_{2}\right]\left(\mathrm{PF}_{6}\right)$ compounds are of the order of $\beta=$ $\pm 5 \times 10^{-30} \mathrm{~cm}^{5} \mathrm{esu}^{-1}$, depending on the substituent $R\left(\mathrm{NH}_{2}\right.$, $\mathrm{H}$ or $\mathrm{NO}_{2}$ ). Sakaguchi et al. (1989) reported values of $\beta=$ $70 \times 10^{-30} \mathrm{~cm}^{5} \mathrm{esu}^{-1}$ for derivatives of $\left[\mathrm{Ru}\left(\operatorname{tris}\left(2,2^{\prime}\right.\right.\right.$-bipyridine))] complexes. It is therefore reasonable to assume $\beta$ values of the order of $10 \times 10^{-30} \mathrm{~cm}^{5} \mathrm{esu}^{-1}$ for trans$\left[\mathrm{RuNO}\left(\mathrm{NH}_{3}\right)_{4} \mathrm{~F}\right] \mathrm{SiF}_{6}$. Based on this assumption, we can rationalize the observed $\mathrm{SH}$ response compared with $\mathrm{LiNbO}_{3}$. $\mathrm{LiNbO}_{3}$ exhibits a $\beta$ value of $21 \times 10^{-24} \mathrm{~cm}^{5} \mathrm{esu}^{-1}$ for particle diameters of $120 \mathrm{~nm}$ (Staedler et al., 2012), which can be related to an average bulk nonlinear coefficient $\langle d\rangle=$ $4.8 \mathrm{pm} \mathrm{V}^{-1}$, where $\langle d\rangle=\beta / V_{\mathrm{p}}$ with $V_{\mathrm{p}}$ the volume of the (nano)particle (Le Dantec et al., 2011). Despite the considerable difference of six orders of magnitude between these
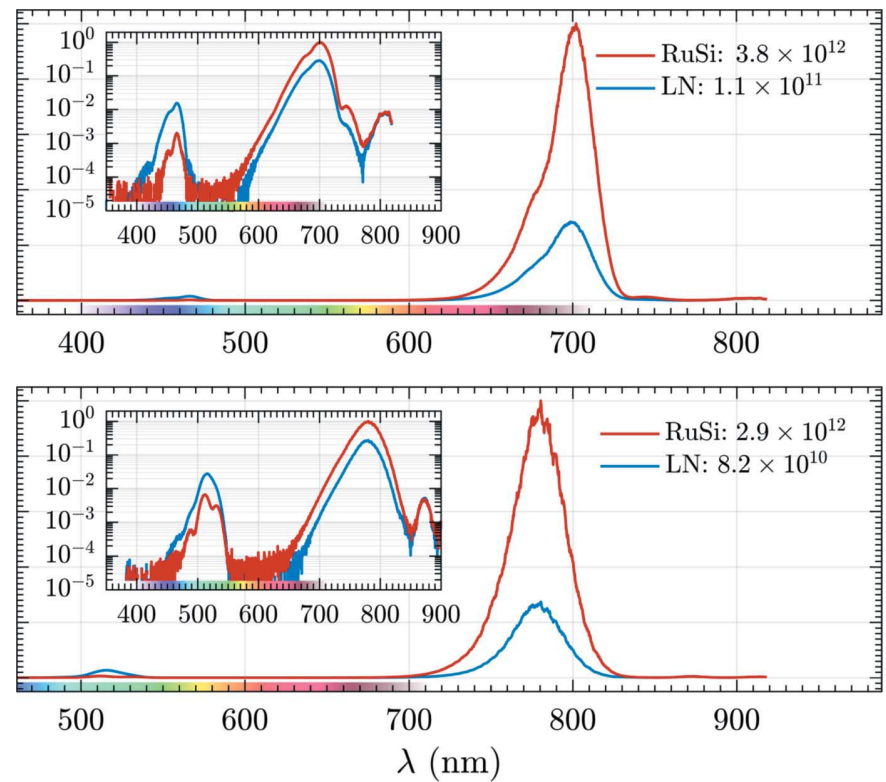

Figure 7

Harmonic emission spectra normalized to the maximum signal of trans$\left[\mathrm{RuNO}\left(\mathrm{NH}_{3}\right)_{4} \mathrm{~F}\right] \mathrm{SiF}_{6}(\mathrm{RuSi})$ and $\mathrm{LiNbO}_{3}: \mathrm{Mg}(\mathrm{LN})$ at fundamental central wavelengths of (top) $\lambda=1400 \mathrm{~nm}$ and (bottom) $\lambda=1560 \mathrm{~nm}$. The insets display the same spectra on a logarithmic scale for better visibility of the third harmonic. Numerical values indicate the harmonic ratio $f_{\mathrm{R}}$. 
hyperpolarizabilities, the differing particle sizes for both materials may easily remedy the observed SH intensity difference which scales with the sixth power of the particle diameter (Kim et al., 2013; Hsieh et al., 2009). In fact, inspection of the prepared trans- $\left[\mathrm{RuNO}\left(\mathrm{NH}_{3}\right)_{4} \mathrm{~F}\right] \mathrm{SiF}_{6}$ powder by optical microscopy shows particles with sizes between a few hundred nanometres and a few micrometres in diameter, thereby compensating for the lower $\beta$ and improving the $\mathrm{SH}$ emission over LN nanoparticles.

Because of MS1 formation at room temperature after bluelight exposure, the possibility of changes in the harmonic emission intensity was investigated next. Furthermore, a timeresolved scan of the nonlinear emission for several minutes allows an assessment in terms of the photostability of the compound in the context of further applications. A selection of such measurements at a fundamental wavelength $\lambda=$ $1560 \mathrm{~nm}$ is presented in Fig. 8.

Exposure at $\mathrm{CW}$ equivalent intensities of $1 \mathrm{~W} \mathrm{~cm}^{-2}$ (bluelight $405 \mathrm{~nm}, \mathrm{CW}$ pump) and $0.8 \mathrm{~W} \mathrm{~cm}^{-2}$ (IR $1560 \mathrm{~nm}$, femtosecond laser) yields a notable decrease in the $\mathrm{SH}$ intensity after a few minutes (see Fig. 8, top). Visual inspection after exposure reveals a considerable darkening of the sample surface, correlating with the significant SH signal decrease, especially during $\mathrm{CW}$ exposure, over time. Because of the pronounced UV/blue absorption band of the complex, a significant amount of energy is deposited in the sample, which exceeds heat-dissipation rates, thereby leading to irreversible
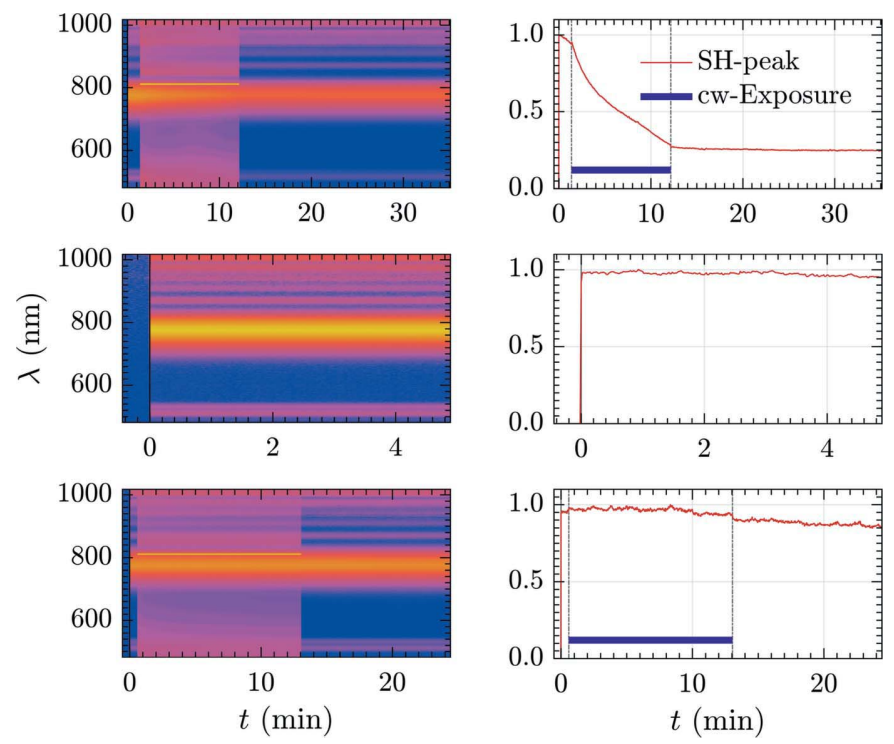

Figure 8

The evolution of (left) the emission spectrum and (right) the normalized emission intensity of the $\mathrm{SH}$ peak as a function of time. Sections of $\mathrm{CW}$ pump exposure in the right-hand plots have been marked. (Top) The emission at the maximum intensity of both IR and blue-light sources. (Middle) The medium intensity of the femtosecond laser source without the pump laser. (Bottom) The emission at medium intensity of both femtosecond and CW lasers. The sharp line visible in the top and bottom spectrotemporal plots at $810 \mathrm{~nm}$ is an artefact originating from the second-order diffraction signal of the pump laser. Similarly, the broadband signal appearing during the pumping process results from stray light from the $\mathrm{CW}$ laser inside the spectrometer. thermal damage. In fact, this behaviour is present even upon femtosecond pulse illumination, as shown for times $t \leq 85 \mathrm{~s}$. Potential causes include a non-negligible absorption at the fundamental wavelength or considerable absorption at the harmonics' wavelengths (i.e. $780 \mathrm{~nm}$ for the second harmonic and $520 \mathrm{~nm}$ for the third harmonic, respectively), as evident in Fig. 4.

As a consequence, the femtosecond IR pulse beam was attenuated to a level where temporally stable, yet significant, harmonic emission was observed for several minutes, as shown in Fig. 8 (middle), resulting in a mean power of $0.5 \mathrm{~W} \mathrm{~cm}^{-2}$ (peak intensity $2 \times 10^{14} \mathrm{~W} \mathrm{~m}^{-2}$ ). At this rate, harmonic emission can easily be detected for a prolonged duration without any notable decrease in the conversion efficiency that would directly correlate with a darkening of the sample surface. Outside of the present investigations, this finding defines an upper limit in disparate areas of application, in particular where the ruthenium complex may be used as a contrast-enhancing agent. Naturally, providing there is sufficient external heat dissipation, a higher $\mathrm{CW}$-equivalent intensity may be attained at no cost of material quality degradation. Vice versa, lower $\mathrm{CW}$-equivalent intensities probably do not harm the sample at all; because of the (peak) intensity dependence of NLO effects on the fundamental wave, a reduction in the pulse repetition rate gives no decrease in the signal intensity per pulse. Thus, NLO emission can be further boosted by focusing, without increasing the radiant exposure by increased pulse-to-pulse delays.

A temporal scan of the $\mathrm{SH}$ emission upon population of MS1 by the CW laser at a lower intensity $\left(0.1 \mathrm{~W} \mathrm{~cm}^{-2}\right)$ reveals a decrease in the nonlinear emission signal after pumping. However, thermal degradation due to the double exposure cannot be fully ruled out, as a slight darkening of the excitation spot is visible at the end of the series. Therefore, a definite answer with regard to a change in polarity upon switching from the ground to the metastable state cannot yet be provided using this method. The strength of such a signal change is determined decisively by both the level of metastable state population and the actual variation in the molecular hyperpolarizability $\beta$, which also depends on the nonnitrosyl ligands of the ruthenium complex and especially their donor-acceptor capability (Akl et al., 2013).

Nevertheless, due to its strong $\mathrm{SH}$ emission, trans$\left[\mathrm{RuNO}\left(\mathrm{NH}_{3}\right)_{4} \mathrm{~F}\right] \mathrm{SiF}_{6}$ may be used in promising applications as a nonlinear emitter. Due to its low water solubility in particular, it is a potential candidate for (biomedical) imaging or detection.

\section{Conclusions}

Long-term treatment of the trans-[RuNO $\left.\left(\mathrm{NH}_{3}\right)_{4} \mathrm{OH}\right] \mathrm{Cl}_{2}$ precursor by $\mathrm{HF}$ allows the quantitative substitution of a hydroxyl ligand by a fluoride, indicating that the substitution reaction is kinetically accessible at $\sim 373 \mathrm{~K}$. Subsequent precipitation of the product by hexafluorosilicate yields the trans-[RuNO$\left.\left(\mathrm{NH}_{3}\right)_{4} \mathrm{~F}\right] \mathrm{SiF}_{6}$ salt, crystallizing in the space group $P n$. Analysis of the non-centrosymmetric structure of 
trans- $\left[\mathrm{RuNO}\left(\mathrm{NH}_{3}\right)_{4} \mathrm{~F}\right] \mathrm{SiF}_{6}$ by Hirshfeld surfaces reveals that $\mathrm{H} \cdots \mathrm{F}$ and $\mathrm{F} \cdots \mathrm{O}$ are the prevalent intermolecular interactions. These interactions result in an almost parallel alignment of the dipole moments of the $\left[\mathrm{RuNO}\left(\mathrm{NH}_{3}\right)_{4} \mathrm{~F}\right]^{2+}$ cations, responsible for the observed NLO properties of the bulk material.

Thermal analysis of the metastable states shows that the increase in the MS2 lifetime leads to a higher probability of the MS2 $\rightarrow$ MS1 reaction, and hence to a higher efficiency of the overall two-step photoisomerization GS $\rightarrow$ MS2 $\rightarrow$ MS1. As a consequence, MS1 can be generated at 290-300 K using a blue-light LED as the irradiation source, thereby opening the possibility for CW-based optical applications at room temperature. On the other hand, the complex shows significant SH emission upon exposure to $1400-1560 \mathrm{~nm}$ femtosecond pulses.

According to the Cambridge Structural Database (Groom et al., 2016), there are at least 100 known octahedral ruthenium nitrosyl complexes which crystallize in a non-centrosymmetric space group. Hence, there are a large number of compounds with potentially interesting NLO properties, in particular SH emission.

Finally, our attempts to modify the emission of the nitrosyl ligand by photoisomerization (GS to MS1) at room temperature were inconclusive. In order to prove SH modification by nitrosyl photoisomerization, the MS1 population needs to be increased, which might be achieved by using other ruthenium nitrosyl compounds and/or working at low temperature.

\section{Acknowledgements}

We thank Sébastien Pillet for help with the calculation of the photodifference maps and Pierrick Durand of the $\mathrm{PMD}^{2} \mathrm{X}$ platform of IJB for performing the powder XRD measurements. We also thank the Federal Agency for Scientific Organizations for funding.

\section{Funding information}

Funding for this research was provided by: Comprehensive Program of Fundamental Research (grant No. 19-03-00594 A to Gennadiy Kostin); Campus France (bursary No. P730329G to Artem Mikhailov); PHC PROCOPE (grant No. 40539XA to Dominik Schaniel); Lorraine Université d'Excellence (grant No. ANR-15-IDEX-04-LUE); CPER (SusChemProc); Deutsche Forschungsgemeinschaft (grant No. DFG INST 190/ 165-1 FUGG to Mirco Imlau); Deutscher Akademischer Austauschdienst (award No. 57390412 to Mirco Imlau).

\section{References}

Akl, J., Billot, C., Lacroix, P. G., Sasaki, I., Mallet-Ladeira, S., Malfant, I., Arcos-Ramos, R., Romero, M. \& Farfán, N. (2013). New J. Chem. 37, 3518-3527.

Asselberghs, I., Clays, K., Persoons, A., Ward, M. D. \& McCleverty, J. (2004). J. Mater. Chem. 14, 2831-2839.

Athmani, H., Kijatkin, C., Benali-Cherif, R., Pillet, S., Schaniel, D., Imlau, M., Benali-Cherif, N. \& Bendeif, E.-E. (2019). Acta Cryst. A75, 107-114.
Bourhis, L. J., Dolomanov, O. V., Gildea, R. J., Howard, J. A. K. \& Puschmann, H. (2015). Acta Cryst. A71, 59-75.

Chemla, D. S. (1980). Rep. Prog. Phys. 43, 1191-1262.

Clays, K. \& Persoons, A. (1991). Phys. Rev. Lett. 66, 2980-2983.

Coe, B. J., Houbrechts, S., Asselberghs, I. \& Persoons, A. (1999). Angew. Chem. Int. Ed. 38, 366-369.

Coppens, P., Novozhilova, I. \& Kovalevsky, A. (2002). Chem. Rev. 102, 861-884.

Cormary, B., Malfant, I., Buron-Le Cointe, M., Toupet, L., Delley, B., Schaniel, D., Mockus, N., Woike, T., Fejfarová, K., Petrícek, V. \& Dusek, M. (2009). Acta Cryst. B65, 612-623.

Dolomanov, O. V., Bourhis, L. J., Gildea, R. J., Howard, J. A. K. \& Puschmann, H. (2009). J. Appl. Cryst. 42, 339-341.

Fomitchev, D. V. \& Coppens, P. (1996). Inorg. Chem. 35, 7021-7026.

Groom, C. R., Bruno, I. J., Lightfoot, M. P. \& Ward, S. C. (2016). Acta Cryst. B72, 171-179.

Hirshfeld, F. L. (1977). Theor. Chim. Acta, 44, 129-138.

Hsieh, C.-L., Grange, R., Pu, Y. \& Psaltis, D. (2009). Opt. Express, 17, 2880-2891.

Kijatkin, C., Eggert, J., Bock, S., Berben, D., Oláh, L., Szaller, Z., Kis, Z. \& Imlau, M. (2017). Photonics, 4, 11.

Kim, E., Steinbrück, A., Buscaglia, M. T., Buscaglia, V., Pertsch, T. \& Grange, R. (2013). ACS Nano, 7, 5343-5349.

Knabe, B., Buse, K., Assenmacher, W. \& Mader, W. (2012). Phys. Rev. B Condens. Matter Mater. Phys. 86, 195428.

Kostin, G. A., Borodin, A. O., Mikhailov, A. A., Kuratieva, N. V., Kolesov, B. A., Pishchur, D. P., Woike, T. \& Schaniel, D. (2015). Eur. J. Inorg. Chem. 2015, 4905-4913.

Kostin, G. A., Mikhailov, A. A., Kuratieva, N. V., Pischur, D. P., Zharkov, D. O. \& Grin, I. R. (2017). New J. Chem. 41, 77587765.

Kostin, G. A., Mikhailov, A. A., Kuratieva, N. V., Pishchur, D. P. \& Makhinya, A. N. (2018). New J. Chem. 42, 18928-18934.

Le Dantec, R., Mugnier, Y., Djanta, G., Bonacina, L., Extermann, J., Badie, L., Joulaud, C., Gerrmann, M., Rytz, D., Wolf, J. P. \& Galez, C. (2011). J. Phys. Chem. C, 115, 15140-15146.

Mikhailov, A. A., Wenger, E., Kostin, G. A. \& Schaniel, D. (2019). Chem. Eur. J. 25, 7569-7574.

Morioka, Y., Ishikawa, A., Tomizawa, H. \& Miki, E. (2000). J. Chem. Soc. Dalton Trans. pp. 781-786.

Ookubo, K., Morioka, Y., Tomizawa, H. \& Miki, E. (1996). J. Mol. Struct. 379, 241-247.

Powell, C. E., Cifuentes, M. P., Morrall, J. P., Stranger, R., Humphrey, M. G., Samoc, M., Luther-Davies, B. \& Heath, G. A. (2003). J. Am. Chem. Soc. 125, 602-610.

Roke, S. \& Gonella, G. (2012). Annu. Rev. Phys. Chem. 63, $353-$ 378.

Sakaguchi, H., Nakamura, H., Nagamura, T., Ogawa, T. \& Matsuo, T. (1989). Chem. Lett. 18, 1715-1718.

Schaniel, D., Bendeif, E.-E., Woike, T., Böttcher, H.-C. \& Pillet, S. (2018). CrystEngComm, 20, 7100-7108.

Schaniel, D., Cormary, B., Malfant, I., Valade, L., Woike, T., Delley, B., Krämer, K. W. \& Güdel, H. U. (2007). Phys. Chem. Chem. Phys. 9, 3717-3724.

Schaniel, D., Imlau, M., Weisemoeller, T., Woike, T., Krämer, K. W. \& Güdel, H.-U. (2007). Adv. Mater. 19, 723-726.

Schaniel, D., Woike, T., Delley, B., Boskovic, C., Biner, D., Krämer, K. W. \& Güdel, H. U. (2005). Phys. Chem. Chem. Phys. 7, 11641170.

Sinitsyn, M. N., Svetlov, A. A., Kanishcheva, A. S., Mikhailov, Y. N., Sadikov, G. G. \& Kokunov, Y. V. B. Y. A. (1989). Zh. Neorg. Khim. 34, 2795-2802.

Spackman, M. A. \& Jayatilaka, D. (2009). CrystEngComm, 11, 19-32. Staedler, D., Magouroux, T., Hadji, R., Joulaud, C., Extermann, J., Schwung, S., Passemard, S., Kasparian, C., Clarke, G., Gerrmann, M., Le Dantec, R., Mugnier, Y., Rytz, D., Ciepielewski, D., Galez, C., Gerber-Lemaire, S., Juillerat-Jeanneret, L., Bonacina, L. \& Wolf, J.-P. (2012). ACS Nano, 6, 2542-2549. 
Su, J., Zhang, J., Tian, X., Zhao, M., Song, T., Yu, J., Cui, Y., Qian, G., Zhong, H., Luo, L., Zhang, Y., Wang, C., Li, S., Yang, J., Zhou, H., Wu, J. \& Tian, Y. (2017). J. Mater. Chem. B, 5, 5458-5463.

Turner, M. J., McKinnon, J. J., Wolff, S. K., Grimwood, D. J., Spackman, P. R., Jayatilaka, D. \& Spackman, M. A. (2017). Crystal Explorer17. University of Western Australia, Australia. http:// hirshfeldsurface.net.
Urban, B. E., Neogi, P., Senthilkumar, K., Rajpurohit, S. K., Jagadeeshwaran, P., Kim, S., Fujita, Y. \& Neogi, A. (2012). IEEE J. Sel. Top. Quantum Electron. 18, 1451-1456.

Weidemann, M., Sievertsen, S. \& Homborg, H. (1998). Z. Anorg. Allg. Chem. 624, 909-918.

Yamaletdinov, R. D. \& Zilberberg, I. L. (2017). Eur. J. Inorg. Chem. 2017, 2951-2954. 